\title{
Lipoproteins and Apolipoproteins in Alzheimer's Disease
}

\author{
Etsuro Matsubara
}

Additional information is available at the end of the chapter

http://dx.doi.org/10.5772/45977

\section{Introduction}

Alzheimer's disease (AD) represents the so-called "storage disorder" of amyloid $\beta(A \beta)$. The $\mathrm{AD}$ brain contains soluble and insoluble $\mathrm{A} \beta$, both of which have been hypothesized to underlie the development of cognitive deficits or dementia (1-3). The steady-state level of $A \beta$ is controlled by the generation of $A \beta$ from its precursor, the degradation of $A \beta$ within the brain, and transport of $A \beta$ out of the brain. The imbalance among three metabolic pathways results in excessive accumulation and deposition of $\mathrm{A} \beta$ in the brain, which may trigger a complex downstream cascade (e.g., primary amyloid plaque formation or secondary tauopathy and neurodegeneration) leading to memory loss or dementia in AD. Accumulated lines of evidence indicate that such a memory loss represents a synaptic failure caused directly by soluble $A \beta$ oligomers (A $\beta \mathrm{Os})$ (4-6), whereas amyloid fibrils may cause neuronal injury indirectly via microglial activation (7). Many attentions are paid to understand the mechanism underlying the neurotoxic action of $\mathrm{A} \beta \mathrm{O}$ s so far. However, the exact metabolic conditions controlling the in vivo generation of soluble $\mathrm{A} \beta \mathrm{O}$ s has been out of attention.

Several lines of evidence indicated that lipidic environments in the central nervous system (CNS) represent one of the prevailing metabolic conditions. We then hypothesized that an alteration of the lipoprotein-soluble $A \beta$ interaction in the CNS is capable of initiating and/or accelerating the cascade favoring $A \beta$ assembly (8). We found that dissociation of $A \beta 42$ from lipoprotein in the cerebrospinal fluid from AD accelerates A $\beta 42$ assembly (9). Thus, lipoprotein is a key molecule to maintain monomeric soluble A $\beta 42$ in CNS.

In this chapter, we review the issue regarding how lipoprotein and apolipoproteins contribute to physiological metabolic conditions. Then, we focus on how they constitute the 
AD-related metabolic conditions in the CNS. We are certain that these points of view introduce a novel approach to find a therapeutic intervention for AD.

\section{Lipoproteins, apolipoproteins, and $\mathrm{A} \beta$ metabolism in the CNS}

In the CNS, we need to be aware that cholesterol metabolism is quite different from that in systemic circulation. Lipidic environments in the CNS were regulated by HDL-like lipoproteins, mainly lipidated apolipoprotein E (apoE), which is in charge of cholesterol transport to and from neurons $(10,11)$. This is also the case in lipidated apolipoprotein J (apoJ) (12). In addition to lipid trafficking, apoE or apoJ as a form of HDL-like lipoprotein plays a major role in $\mathrm{A} \beta$ metabolism in the CNS. Both apolipoproteins are well known as major carrier proteins for $\mathrm{A} \beta$ (13-17). Interestingly, transgenic mouse models of $\mathrm{AD}$ (apoE$1-/$ apoJ ${ }^{-/-}$) revealed that both apolipoproteins regulate in a cooperative manner the clearance and the deposition of $A \beta$ in brain (18). The hypothetical pathways involved in the clearance of $C N S A ß$ are efflux of $A ß$ into the plasma via blood-brain barrier (BBB). Two lipoprotein-receptors, LRP-1 and LRP-2, seem to be responsible for efflux of lipoprotein-free or lipoprotein-associated (apoJ-associated) Aß from the brain to blood, respectively (19). In vivo relevance of LRP-1-mediated Aß transport has been confirmed in transgenic mice expressing low LRP-1-receptor and APP, which develops extensive Aß accumulation much faster than transgenic mice expressing high level of APP (20). Reduced expression of brain endotherial LRP-1 was also observed in AD patients, which was associated with impaired $A ß$ clearance and cerebrovascular accumulation. LPR-2 appeared to function bi-directionally (influx vs efflux) at BBB. In contrast to LRP2mediated influx (21), LPR2-mediated efflux of brain Aß was actively operated under physiological concentration of either $A ß$ or apoJ (19). Interestingly, a recent study shows that apoE4 binding to $A B$ redirects its clearance from LRP-1 to VLDLR, which resulted in slower efflux of brain Aß than LRP-1 (22). In contrast, apoE2-Aß and apoE3-Aß complexes are cleared at BBB via both LPR-1 and VLDLR at a substantially faster rate than apoE4-Aß complexes(22). Impairment of the above-mentioned receptor-mediated clearance at BBB could contribute to the pathogenesis of AD. Alternatively, ApoE4-HDL shows less cholesterol exchange between lipid particles and the neuronal membrane as compared with apoE3-HDL (23), leading to altered membrane functions, e.g., signal transduction, enzyme activities, ion channel properties, and conformation of sAß peptides, which contribute to the disease-related metabolic conditions. Furthermore, when the generation of HDL-like lipoproteins in the AD mouse model is suppressed or overexpressed via the specific regulation of ATP-binding cassette A1 (ABCA1), A $\beta$ deposition exhibits augmentation or reduction, respectively, which depends on the degree of ABCA1mediated lipidation of apoE in the CNS $(24,25)$. From these points of view, lipidic environments in the CNS represent one of the prevailing metabolic conditions. We hypothesized that an alteration of the lipoprotein-sA $\beta$ interaction in the CNS is capable of initiating and/or accelerating the cascade favoring $A ß$ assembly. Thus, we postulate that 
lipoproteins or apolipoproteins may regulate the metabolic conditions controlling the in vivo generation of soluble $\mathrm{A} \beta \mathrm{O}$.

\section{Aß is present in either lipoprotein-free or lipoprotein-associated form in brain parenchyma}

To assess the above-mentioned issue, we examined whether the dissociation of sAß from lipoprotein-particles occurs in the brain. The combination of size exclusion chromatography (SEC) and enzyme-linked immunosorbent assay (ELISA) revealed that the dissociation of $\mathrm{SA}$ from lipoprotein-particles occurs in brain parenchyma and the presence of soluble dimeric lipoprotein-free $A ß$ in $A D$ brains (8). These findings may support the hypothesis that functionally declined lipoproteins may be major determinants in the production of metabolic conditions leading to higher levels of soluble dimeric SDS-resistant form of A $\beta$ in $\mathrm{AD}$ brains $(8,26)$. At this moment, it remains undetermined whether dissociation of $\mathrm{A} \beta$ from lipoprotein or less association of $A \beta$ to lipoproteins accounts for such a metabolic conditions. To further verify this hypothesis, we focused on the entorhinal cortex (EC), followed by biochemical analyses using an anti-oligomer specific antibody, namely 2C3 (9, 27). Fifty brains obtained from healthy elderly are composed of three Braak NFT stages; Braak NFT stages I-II ( $n=35$, normal control); Braak NFT stages III-IV ( $n=13$, MCI stage); Braak NFT stages IV-V ( $n=2$, AD stages). Immunoblot analysis of the delipidated EC employing monoclonal $2 \mathrm{C} 3$ revealed that the accumulation of soluble 12-mers precedes the appearance of neuronal loss or cognitive impairment, and is enhanced as the Braak neurofibrially tangle (NFT) stages progress, indicating that the ECs of AD patients indeed bear metabolic conditions that accelerate $\mathrm{A} \beta$ assembly.

\section{4. $A ß$ is present in either lipoprotein-free or lipoprotein-associated form in cerebrospinal fluid (CSF) ${ }^{9}$}

The presence of lipoprotein-free sA $\beta O$ s in CSF was also assessed in age-matched normal controls (NCs) and patients with Alzheimer's disease (AD) by SEC and ELISA specific for either $\mathrm{A} \beta \mathrm{Os}$ or $\mathrm{A} \beta \mathrm{Ms}$. The SEC experiment using pooled CSF revealed that the dissociation of $\mathrm{sA} \beta \mathrm{Ms}$ from lipoprotein particles indeed occurs in CSF, which was lower in AD than in NCs. Furthermore, the SEC experiment using lipoprotein-depleted pooled CSF (LPD-CSF) confirmed the presence of oligomeric $2 \mathrm{C} 3$ conformers (4- to 35-mers), which appeared to be higher in AD patients than in NCs. To address the issue on the presence of any metabolic conditions favoring $A \beta$ assembly, we compared the levels of lipoprotein-free sA $\beta M$ s and $\mathrm{sA} \beta \mathrm{O}$ s in LPD-CSF from the 12 sporadic AD patients and $13 \mathrm{NCs}$ to evaluate the A $\beta \mathrm{Os} /$ $\mathrm{A} \beta \mathrm{Ms}$ ratio (the $\mathrm{O} / \mathrm{M}$ index). The levels of $2 \mathrm{C} 3$ oligomeric conformers composed of $\mathrm{A} \beta 42$ are significantly higher in AD patients than in NCs. The O/M index for either $A \beta 42$ or $A \beta 40$ is also significantly higher in AD patients than in NCs. Of note, the relative amounts of total lipoprotein-associated sA $\beta$ Ms ( 70\%) versus lipoprotein-free sA $\beta$ Ms $(\sim 30 \%)$ remained 


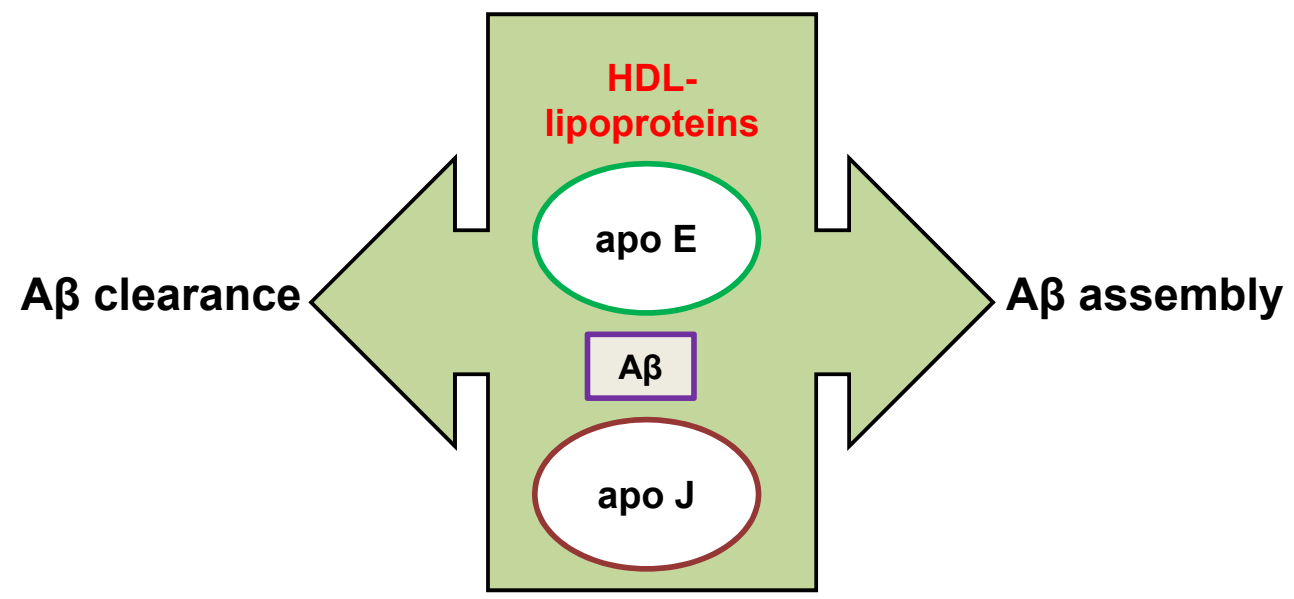

Figure 1. Hypothetical metabolic conditions favoring $\mathrm{A} \beta$ assembly. Functionally declined lipoproteins may accelerate the generation of metabolic conditions leading to higher levels of soluble A $\beta$ assembly in the CNS. 
essentially unchanged in sporadic AD patients as compared with NCs. However, the relative amounts of lipoprotein-free $\mathrm{A} \beta 42$ was significantly lower in the sporadic $\mathrm{AD}$ patients $(9.3 \pm 3.9 \%)$ than in NCs $(13.2 \pm 4.5 \%)$, which is in accordance with our abovementioned finding that the level of oligomeric $2 \mathrm{C} 3$ conformers composed of $\mathrm{A} \beta 42$ was significantly elevated in AD patients. Thus, it is likely that the conversion of lipoprotein-free monomeric soluble A $\beta 42$ into oligomeric assembly preferentially occurs in AD CSF, mirroring the disease-related metabolic conditions in the brain parenchyma.

\section{Summary}

We previously reported that $\sim 90 \%$ of sA $\beta \mathrm{Ms}$ that circulate in normal plasma is associated with lipoprotein particles (27). From the above data, it is plausible to assume that about $70 \%$ of CSF sA $\beta \mathrm{Ms}$ is normally associated with lipoprotein particles, indicating that CNS constitutes a risky environment where the lipoproteins-sA $\beta$ Ms interaction is impaired, leading to $A \beta$ assembly. From this point of view, a key molecule to maintain monomeric sA 342 metabolism in CNS appears to be HDL-like lipoprotein particles. In this sense, the dissociation of sA $\beta 42$ from or the lack of association with HDL-like lipoprotein particles not only constitutes a potential mechanism to initiate and/or accelerate the cascade favoring A $\beta 42$ assembly in the brain, but also results in a reduced clearance of physiological lipoprotein-associated sA $\beta 42$ peptides in the brain. Thus, above-mentioned CNS environments may strongly affect conformation of $\mathrm{sA} \beta$ peptides, resulting in the conversion of sA $\beta 42$ monomers into sA $\beta 42$ assembly. The findings suggest that functionally declined lipoproteins may accelerate the generation of metabolic conditions leading to higher levels of sA $\beta 42$ assembly in the CNS.

\section{Author details}

Etsuro Matsubara

Department of Neurology, Institute of Brain Science, Hirosaki Graduate School of Medicine, Japan

\section{References}

[1] Hardy J, Allsop D: Amyloid deposition as the central event in the aetiology of Alzheimer's disease. Trends Pharmacol Sci 1991, 12: 383-388.

[2] Lue LF, Kuo YM, Roher AE, Brachova L, Shen Y, Sue L, Beach T, Kurth JH, Rydel RE, Rogers J: Soluble amyloid $\beta$ peptide concentration as a predictor of synaptic change in Alzheimer's disease. Am J Pathol 1999, 155: 853-862.

[3] McLean CA, Cherny RA, Fraser FW, Fuller SJ, Smith MJ, Beyreuther K, Bush AI, Masters CL: Soluble pool of Abeta amyloid as a determinant of severity of neurodegeneration in Alzheimer's disease. Ann Neurol 1999, 46: 860-866. 
[4] Klein WL, Krafft GA, Finch CE: Targeting small Abeta oligomers: the solution to an Alzheimer's disease conundrum? Trends Neurosci 2001, 24: 219-224.

[5] Selkoe DJ: Alzheimer's disease is a synaptic failure. Science 2002, 298: 789-791.

[6] Hass C, SelkoeDJ: Soluble protein oligomers in neurodegeneration: lessons from the Alzheimer's amyloid $\beta$-peptide. Nat Rev Mol Cell Biol 2007, 8: 101-112.

[7] Akiyama H, Barger S, Barnum S, Bradt B, Bauer J, Cole GM, Cooper NR, Eikelenboom P, Emmerling M, Fiebich BL, Finch CE, Frautschy S, Griffin WS, Hampel H, Hull M, Landreth G, Lue L, Mrak R, Mackenzie IR, McGeer PL, O'Banion MK, Pachter J, Pasinetti G, Plata-Salaman C, Rogers J, Rydel R, Shen Y, Streit W, Strohmeyer R, Tooyoma I, Van Muiswinkel FL, Veerhuis R, Walker D, Webster S, Wegrzyniak B, Wenk G, Wyss-Coray T: Inflammation and Alzheimer's disease. Neurobiol Aging 2000, 21: 383-421.

[8] Matsubara E, Sekijima Y, Tokuda T, Urakami K, Amari M, Shizuka-Ikeda M, Tomidokoro Y, Ikeda M, Kawarabayashi T, Harigaya Y, Ikeda S, Murakami T, Abe K, Otomo E, Hirai S, Frangione B, Ghiso J, Shoji M. 2004. Soluble Abeta homeostasis in AD and DS: impairment of anti-amyloidogenic protection by lipoproteins. Neurobiol Aging 25:833-841.

[9] Takamura A, Kawarabayashi T, Yokoseki T, Shibata M, Morishima-Kawashima M, Saito Y, Murayama S, Ihara Y, Abe K, Shoji M, Michikawa M, Matsubara E. The Dissociation of $\mathrm{A} \beta$ from Lipoprotein in Cerebrospinal Fluid from Alzheimer's Disease accelerates Aß42 assembly. J Neurosci Res. 2011;89(6):815-821.

[10] Michikawa M, Gong JS, Fan QW, Sawamura N, Yanagisawa K. 2001. A novel action of alzheimer's amyloid beta-protein (Abeta): oligomeric Abeta promotes lipid release. J Neurosci 21:7226-7235.

[11] Gong JS, Sawamura N, Zou K, Sakai J, Yanagisawa K, Michikawa M. 2002. Amyloid beta-protein affects cholesterol metabolism in cultured neurons: implications for pivotal role of cholesterol in the amyloid cascade. J Neurosci Res 70:438-46.

[12] DeMattos RB, Brendza RP, Heuser JE, Kierson M, Cirrito JR, Fryer J, Sullivan PM, Fagan AM, Han X, Holtzman DM. Purification and characterization of astrocyte-secreted apolipoprotein $\mathrm{E}$ and J-containing lipoproteins from wild-type and human apoE transgenic mice. Neurochem Int. 2001, 39(5-6):415-25.

[13] Ghiso J, Matsubara E, Koudinov A, Choi-Miura NH, Tomita M, Wisniewski T, Frangione B. The cerebrospinal-fluid soluble form of Alzheimer's amyloid $B$ is complexed to SP-40,40 (apolipoprotein J), an inhbitor of the complement membraneattack complex. Biochem J.,1993;293:27-30.

[14] Wisniewski T, Golabek A, Matsubara E, Ghiso J, Frangione B. Apolipoprotein E: binding to soluble Alzheimer's B -amyloid. Biochem Biophys Res Commun., 1993;192:359-365.

[15] Koudinov A, Matsubara E, Frangione B, Ghiso J. The soluble form of Alzheimer's amyloid $B$ protein is complexed to high density lipoprotein 3 and very high density 
lipoprotein in normal human plasma. Biochem Biophys Res Commun., 1994;205:11641171.

[16] Matsubara E, Frangione B, Ghiso J. Characterization of apolipoprotein J-Alzheimer's A beta interaction. J Biol Chem. 1995, 270:7563-7567.

[17] Matsubara E, Soto C, Governale S, Frangione B, Ghisom J. Apolipoprotein J and Alzheimer's amyloid B solubility. Biochem J, 1996;316:671-679.

[18] DeMattos RB, Bales KR, Cummins DJ, Paul SM, Holtzman DM. Brain to plasma amyloid-beta efflux: a measure of brain amyloid burden in a mouse model of Alzheimer's disease. Science 295: 2264-2267, 2002

[19] Bell RD, Sagare AP, Friedman AE, Bedi GS, Holtzman DM, Deane R, Zlokovic BV. Transport pathways for clearance of human Alzheimer's amyloid beta-peptide and apolipoproteins $\mathrm{E}$ and $\mathrm{J}$ in the mouse central nervous system. J Cereb Blood Flow Metab. 27:909-918, 2007.

[20] Shibata M, Yamada S, Kumar SR, Calero M, Bading J, Frangione B, Holtzman DM, Miller CA, Strickland DK, Ghiso J, Zlokovic BV. Clearance of Alzheimer's amyloid-ss(140) peptide from brain by LDL receptor-related protein-1 at the blood-brain barrier. J Clin Invest. 2000 Dec;106(12):1489-99.

[21] Shayo M, McLay RN, Kastin AJ, Banks WA. The putative blood-brain barrier transporter for the beta-amyloid binding protein apolipoprotein $\mathrm{J}$ is saturated at physiological concentration. Life Sci 60: 115-118, 1997.

[22] Deane R, Sagare A, Hamm K, Parisi M, Lane S, Finn MB, Holtzman DM, Zlokovic BV. apoE isoform-specific disruption of amyloid beta peptide clearance from mouse brain. J Clin Invest. 2008 Dec;118(12):4002-13.

[23] Zou K, Gong JS, Yanagisawa K, Michikawa M. 2002. A novel function of monomeric amyloid beta-protein serving as an antioxidant molecule against metal-induced oxidative damage. J Neurosci 22:4833-4841.

[24] Wahrle SE, Jiang H, Parsadanian M, Hartman RE, Bales KR, Paul SM, Holtzman DM. 2005. Deletion of Abca1 increases Abeta deposition in the PDAPP transgenic mouse model of Alzheimer disease. J Biol Chem 280:43236-43242.

[25] Wahrle SE, Jiang H, Parsadanian M, Kim J, Li A, Knoten A, Jain S, Hirsch-Reinshagen V, Wellington CL, Bales KR, Paul SM, Holtzman DM. 2008. Overexpression of ABCA1 reduces amyloid deposition in the PDAPP mouse model of Alzheimer disease. J Clin Invest 118:671-682.

[26] Matsubara E, Ghiso J, Frangione B, Amari M, Tomidokoro Y, Ikeda Y, Harigaya Y, Okamoto K, Shoji M. 1999. Lipoprotein-free amyloidogenic peptides in plasma are elevated in patients with sporadic Alzheimer's disease and Down's syndrome.Ann Neurol 45:537-541.

[27] Takamura A, Okamoto Y, Kawarabayashi T, Yokoseki T, Shibata M, Mouri A, Nabeshima T, Sun H, Abe K, Shoji M, Yanagisawa K, Michikawa M, Matsubara E. 
Extracellular and Intraneuronal HMW-AbetaOs Represent a Molecular Basis of Memory Loss in Alzheimer's Disease Model Mouse. Mol Neurodegener. 2011;20: 6. 